Article

\title{
Invariant Solutions for a Class of Perturbed Nonlinear Wave Equations
}

\author{
Waheed A. Ahmed, F. D. Zaman and Khairul Saleh * \\ Department of Mathematics and Statistics, King Fahd University of Petroleum and Minerals, Dhahran 31261, \\ Saudi Arabia; waheed@hotmail.com (W.A.A.); fzaman@kfupm.edu.sa (F.D.Z.) \\ * Correspondence: khairul@kfupm.edu.sa; Tel.: +966-13-8607524
}

Received: 22 September 2017; Accepted: 23 October 2017; Published: 1 November 2017

\begin{abstract}
Approximate symmetries of a class of perturbed nonlinear wave equations are computed using two newly-developed methods. Invariant solutions associated with the approximate symmetries are constructed for both methods. Symmetries and solutions are compared through discussing the advantages and disadvantages of each method.
\end{abstract}

Keywords: approximate symmetry; approximate invariant solution; nonlinear wave equation

Mathematics Subject Classification: 35L05; 35L70; 58J70; 74J30; 76M60

\section{Introduction}

Approximate Lie symmetry is based on the utilization of the perturbation approach in finding symmetries of certain equations. Baikov, Gazizov and Ibragimov [1] proved an approximate Lie theorem enabling one to construct approximate symmetries of differential equations that are stable under small perturbations. Fushchich and Shtelen [2] and later Gazizov [3] introduced approximate symmetries of differential equations with small perturbations and showed that the these symmetries form an approximate Lie algebra. Since then, many authors have used the approximate Lie symmetries to study nonlinear partial differential equations (PDEs) with a small parameter; see, for instance, [4-7] and the references therein.

Pakdermirli, Yurusoy and Dolapci [8] provided a comparison between several methods that use approximate symmetries. Valenti [9] calculated the solution of a model describing dissipative media using the generator of the first-order approximate symmetries. Bokhari, Kara and Zaman [4] considered some nonlinear evolution equations with a small parameter and their symmetries. On the other hand, a refined invariant subspace method to determine subspaces of solutions to nonlinear wave equations was discussed in [10]. Zhi-Yong, Yu-Fu and Xue-Lin [11] performed classification and gave approximate solutions to a class of perturbed nonlinear wave equation employing the method originated from Fushchich and Shtelen. In [12], the authors introduced a new method to obtain the approximate symmetry of the nonlinear evolution equation from perturbations.

In this paper, we study the approximate symmetries of a class of perturbed nonlinear wave equations given by:

$$
u_{t t}+\alpha u_{t}=\left(g(u) u_{x}\right)_{x}+\left(h(u) u_{y}\right)_{y}+\beta f(u) .
$$

Lie group theory provides a systematic way of finding exact solutions of differential equations. If the problem involves a small parameter, then an approximate solution instead of an exact solution can be sought. We employ two methods in which a combination of Lie symmetries and perturbation theory is used to find approximate Lie symmetries and invariant solutions.

Method I was introduced by Baikov, Gazizov and Ibragimov [1,13]. In this method, an approximate generator is calculated to obtain the solution. The Lie operator is expanded in a 
perturbation series other than perturbation for dependent variables as in the usual case. In other words, it is assumed that the perturbed differential equation is of the form:

$$
F(z)=F_{0}(z)+\varepsilon F_{1}(z)=0,
$$

where $z=(x, u, u(1), \ldots, u(n)), F_{0}(z)=0$ is the unperturbed equation and $F_{1}(z)$ is the perturbed term.

Theorem 1. [14] Equation (2) is approximately invariant with the generator $X=X^{0}+\varepsilon X^{1}$ if and only if:

$$
[X F]_{F \approx 0}=O(\varepsilon) \quad \text { or } \quad\left[X^{0} F^{0}+\varepsilon\left(X^{1} F_{0}+X^{0} F_{1}\right]_{F \approx 0}=O(\varepsilon),\right.
$$

in which $X^{0}$ is a generator of Lie symmetry of $F_{0}=0$ and $X^{1}$ is a generator of Lie symmetry of $F_{1}$.

The exact symmetry of the unperturbed equation $F_{0}(z)=0$ denoted by $X^{0}$ can be obtained using the equation $\left.X^{0} F_{0}(z)\right|_{F_{0}(z)=0}=0$. Applying the auxiliary function:

$$
H=\left.\frac{1}{\varepsilon} X^{0}\left(F_{0}(z)+\varepsilon F_{1}(z)\right)\right|_{F 0+e F 1=0}
$$

we deduce the vector field $X^{1}$ from the relation:

$$
\left.X^{1} F_{0}(z)\right|_{F 0=0}+H=0 .
$$

After computing the approximate symmetries, the corresponding invariant solutions are constructed via the classical Lie symmetry method [14]. One may refer the reader for some cases of studying unperturbed and perturbed non-linear wave equations to Bokhari, Kara, Karim, Zaman [15] and Zhi-Yong, Yu-Fu and Xue-Lin [12]. Ahmed, Bokhari, Kara and Zaman [16] provided a classification of the symmetries of the unperturbed nonlinear $(2+1)$ dimensional wave equation with its respective commutator table.

Method II is due to Fushchich and Shtelen [2] and later followed by Euler et al. [17] and Euler and Euler [18]. In this method, the dependent variables are expanded in a perturbation series as is done in the usual perturbation analysis (see, e.g., $[19,20]$ ). The approximate symmetry of the original equation is defined to be the exact symmetry of the coupled equations.

Consider the general $m$-th order nonlinear evolution equation:

$$
E=E\left(x, t, u, u_{1}, u_{2}, \ldots, u_{m}, u_{t} ; \varepsilon\right)=0,
$$

where $u_{t}=\partial u / \partial t, u_{k}=\partial^{k} u / \partial x^{k}, 1 \leq k \leq m, \varepsilon$ is a small parameter and $E$ is a smooth function of the indicated variables. Expanding the dependent variable in the small parameter yields:

$$
u=u_{0}+\varepsilon u_{1}+\ldots, \quad 0<\varepsilon<1 .
$$

Inserting expansion Equation (5) into the original Equation (4) and separating at each order of the perturbed parameter, one has:

$$
\operatorname{Order} \varepsilon^{0}: E_{0}=0, \operatorname{Order} \varepsilon^{1}: E_{1}=0,
$$

and hence, the exact symmetry of system Equation (6) is the approximate symmetry of the original Equation (4).

The outline of this paper is as follows. In Section 2, we construct invariant solutions of a perturbed nonlinear $(1+1)$-dimensional wave equation. In Section 3, we consider Equation (1) with $\beta=0$ and obtain exact and approximate symmetries of the equation using the approximate Lie symmetry Method I. Moreover approximate invariant solutions of the perturbed non-linear wave equation based on the Lie group method are constructed. In Section 4, we discuss Equation (1) with $\alpha=0$ and compute 
approximate symmetries of the equation with a forcing term using both the approximate Lie symmetry methods. We compare these different methods and discuss the advantages of using one over the other. Moreover, approximate invariant solutions of the nonlinear wave equation with a forcing term based on the Lie group method are constructed.

\section{Perturbed Nonlinear (1 + 1)-Dimension Wave Equation}

Consider the perturbed nonlinear wave equation (see e.g., [21]):

$$
F_{0}(z)+\varepsilon F_{1}(z)=u_{t t}-\left(u^{2} u_{x}\right)_{x}+\varepsilon u_{t}=0 .
$$

The approximate group generator of Equation (7) is of the form:

$$
X=X^{0}+\varepsilon X^{1} \equiv\left(\tau_{0}+\varepsilon \tau_{1}\right) \frac{\partial}{\partial t}+\left(\xi_{0}+\varepsilon \xi_{1}\right) \frac{\partial}{\partial x}+\left(\eta_{0}+\varepsilon \eta_{1}\right) \frac{\partial}{\partial u},
$$

where $\tau_{j}, \xi_{j}, \eta_{j}(j=0,1)$ are all unknown functions of $t, x$, and $u$. The infinitesimal generator for the unperturbed equation is a vector field in the three-dimensional space (two independent variables and one dependent variable):

$$
X^{0}=\tau_{0} \frac{\partial}{\partial t}+\xi_{0} \frac{\partial}{\partial x}+\eta_{0} \frac{\partial}{\partial u} .
$$

The prolongation of the infinitesimal symmetry generator is given by:

$$
X^{0^{(2)}}=X^{0}+\eta_{0}^{t} \frac{\partial}{\partial u_{t}}+\eta_{0}^{x} \frac{\partial}{\partial u_{x}}+\eta_{0}^{t t} \frac{\partial}{\partial u_{t t}}+\eta_{0}^{x t} \frac{\partial}{\partial u_{x t}}+\eta_{0}^{x x} \frac{\partial}{\partial u_{x x}} .
$$

The symmetry criterion of Equation (10) yields the relation:

$$
\left.X^{0^{(2)}}\left(u_{t t}-\left(u^{2} u_{x}\right) x\right)\right|_{u_{t t}-\left(u^{2} u_{x}\right)_{x}}=0 .
$$

Comparing coefficients of $u_{x}, u_{x}^{2}, \ldots$, we obtain the following system of determining equations.

$$
\begin{aligned}
& \xi_{0_{u}}=0, \quad \xi_{0_{t}}=0, \quad \tau_{0_{u}}=0, \quad \tau_{0_{x}}=0, \quad \eta_{0_{u u}}=0, \\
& 2 \eta_{0}+2 u \eta_{0_{u}}-4 u \xi_{0_{x}}+4 u \tau_{0_{t}}=0, \quad 2 u^{2} \eta_{0_{x u}}-u \eta_{0_{x x}}+4 u \eta_{0_{x}}=0, \\
& -2 \eta_{0_{t u}}+\tau_{0 t t}=0, \quad 2 u \eta_{0}+2 u^{2} \eta_{0_{x}}+2 u^{2} \tau_{0 t}=0, \quad u^{2} \eta_{0_{x x}}-\eta_{0_{t t}}=0
\end{aligned}
$$

Solving this system of PDEs, we obtain:

$$
\xi_{0}=\alpha_{0}+\alpha_{1} x, \quad \tau_{0}=\alpha_{3} t+\alpha_{1} t+\alpha_{2}, \quad \eta_{0}=-\alpha_{3} u,
$$

where $\alpha_{0}, \alpha_{1}, \alpha_{2}, \alpha_{3}$ are arbitrary constants. Thus,

$$
X^{0}=\left(\alpha_{0}+\alpha_{1} x\right) \frac{\partial}{\partial x}+\left(\alpha_{3} t+\alpha_{1} t+\alpha_{2}\right) \frac{\partial}{\partial t}-\alpha_{3} u \frac{\partial}{\partial u}
$$

To determine the auxiliary function $H$, we consider:

$$
H=\left.\frac{1}{\varepsilon}\left[X^{0^{(2)}}\left[f_{0}(z)+\varepsilon F_{1}(z)\right]\right]\right|_{\left\{F_{0}(z)+\varepsilon F_{1}(z)=0\right\}},
$$

or:

$$
H=\left.\frac{1}{\varepsilon}\left[X^{0^{(2)}}\left[u_{t t}-2 u u_{x}^{2}-u^{2} u_{x x}+\varepsilon u_{t}\right]\right]\right|_{\left\{u_{t t}-2 u u_{x}^{2}-u^{2} u_{x x}+\varepsilon u_{t}=0\right\}},
$$

where $X^{0^{(2)}}$ is the second prolongation of $X^{0}$. This implies that: 


$$
H=\left.\frac{1}{\varepsilon}\left[\eta\left(-2 u_{x}^{2}-2 u u_{x x}\right)+\eta^{x}\left(-4 u u_{x}^{2}\right)+\eta^{t}(\varepsilon)+\eta^{x x}\left(-u^{2}\right)+\eta^{t t}\right]\right|_{\left\{u_{t t}-2 u u_{x}^{2}-u^{2} u_{x x}+\varepsilon u_{t}=0\right\}} .
$$

Hence,

$$
\begin{aligned}
& \eta=-\alpha_{3} u, \quad \eta^{x}=-\alpha_{3} u_{x,} \quad \eta^{t}=\alpha_{4} u_{t}-\alpha_{3} u_{t}, \\
& \eta^{t t}=\alpha_{4} u_{t t}-2 \alpha_{3} u_{t t}, \quad \eta^{x x}=-\alpha_{4} u_{x x}-2 \alpha_{3} u_{x x} .
\end{aligned}
$$

Substituting $\eta, \eta^{x}, \eta^{t}, \eta^{t t}, \eta^{x x}$ and $u_{t t}=2 u u_{x}^{2}+u^{2} u_{x x}-\varepsilon u_{t}$ into Equation (16) gives:

$$
H=\alpha_{3} u_{t}
$$

The determining equation for deformations is written as:

$$
\left.X^{1^{(2)}}\left(u_{t t}-u^{2} u_{x x}-2 u u_{x}^{2}\right)\right|_{u_{t t}=u^{2} u_{x x}+2 u u_{x}^{2}}+H=0,
$$

where $X^{1^{(2)}}$ denotes the second prolongation of the operator:

$$
X^{1}=\tau_{1} \frac{\partial}{\partial t}+\xi_{1} \frac{\partial}{\partial x}+\eta_{1} \frac{\partial}{\partial u} .
$$

We obtain the following system of the determining equations for Equation (18):

$$
\begin{aligned}
& \xi_{1_{u}}=0, \quad \xi_{1_{t}}=0, \quad \tau_{1_{u}}=0, \quad \tau_{1_{x}}=0, \quad \eta_{1_{u u}}=0, \\
& 2 \eta_{1}+2 u \eta_{1_{u}}-4 u \xi_{1_{x}}+4 u \tau_{1_{t}}=0, \quad 2 u^{2} \eta_{1_{x u}}-u \eta_{1_{x x}}+4 u \eta_{1_{x}}=0, \\
& -2 \eta_{1_{t u}}+\tau_{1 t t}-\alpha_{3}=0, \quad 2 u \eta_{1}+2 u^{2} \eta_{1_{x}}+2 u^{2} \tau_{1 t}=0, \quad u^{2} \eta_{1_{x x}}-\eta_{1_{t t}}=0
\end{aligned}
$$

Solving the above system yields:

$$
\tau_{1}=\beta_{1}+\beta_{3} t+\frac{1}{6} \alpha_{3} t^{2}, \quad \xi_{1}=\beta_{2}+\left(\beta_{3}+\beta_{4}\right) x, \quad \eta_{1}=\left(\beta_{4}-\frac{1}{3} \alpha_{3} u\right) u
$$

Substituting Equations (12) and (20) into Equation (8), we obtain the following approximate symmetries for Equation (7):

$$
\begin{array}{r}
X_{1}=\frac{\partial}{\partial t}, \quad X_{2}=\frac{\partial}{\partial x}, \quad X_{3}=t \frac{\partial}{\partial t}+x \frac{\partial}{\partial x}+\frac{\varepsilon}{6}\left(t^{2} \frac{\partial}{\partial t}-2 t u \frac{\partial}{\partial u}\right), \\
X_{4}=x \frac{\partial}{\partial x}+u \frac{\partial}{\partial u}, \quad X_{5}=\varepsilon X_{1}, \quad X_{6}=\varepsilon X_{2}, \quad X_{7}=\varepsilon X_{4}, \quad X_{8}=\varepsilon X_{3} .
\end{array}
$$

In Table 1, we show that the generators span an eight-dimensional approximate Lie algebra and, hence, generate an eight-parameter approximate transformation group.

Table 1. Approximate commutators of approximate symmetry of the perturbed non-linear wave equation.

\begin{tabular}{ccccccccc}
\hline & $\boldsymbol{X}_{\mathbf{1}}$ & $\boldsymbol{X}_{\mathbf{2}}$ & $\boldsymbol{X}_{\mathbf{3}}$ & $\boldsymbol{X}_{\mathbf{4}}$ & $\boldsymbol{X}_{\mathbf{5}}$ & $\boldsymbol{X}_{\mathbf{6}}$ & $\boldsymbol{X}_{\mathbf{7}}$ & $\boldsymbol{X}_{\mathbf{8}}$ \\
\hline$X_{1}$ & 0 & 0 & $X_{1}+\frac{1}{3}\left(X_{8}-X_{7}\right)$ & 0 & 0 & 0 & 0 & $X_{5}$ \\
$X_{2}$ & 0 & 0 & $X_{2}$ & $X_{2}$ & 0 & 0 & $X_{6}$ & $X_{6}$ \\
$X_{3}$ & 0 & 0 & 0 & 0 & $-X_{5}$ & $-X_{6}$ & 0 & 0 \\
$X_{4}$ & 0 & 0 & 0 & 0 & 0 & $-X_{6}$ & 0 & 0 \\
$X_{5}$ & 0 & 0 & $X_{6}$ & $X_{6}$ & 0 & 0 & 0 & 0 \\
$X_{6}$ & 0 & 0 & $X_{6}$ & 0 & 0 & 0 & 0 & 0 \\
$X_{7}$ & 0 & $-X_{6}$ & 0 & 0 & 0 & 0 & 0 & 0 \\
$X_{8}$ & $-X_{5}$ & $-X_{6}$ & 0 & 0 & 0 & 0 & 0 & 0 \\
\hline
\end{tabular}


Approximate Invariant Solution

Using the symmetry $X=X_{3}-X_{4}$, we obtain:

$$
X=t \frac{\partial}{\partial t}-u \frac{\partial}{\partial u}+\frac{\varepsilon}{6}\left(t^{2} \frac{\partial}{\partial t}-2 t u \frac{\partial}{\partial u}\right)
$$

The approximate invariants of Equation (21) can be written as:

$$
E(t, x, u, \varepsilon)=E^{0}(t, x, u)+\varepsilon E^{1}(t, x, u)+O(\varepsilon),
$$

which lead to the system:

$$
\begin{aligned}
& t \frac{\partial E^{0}}{\partial t}-u \frac{\partial E^{0}}{\partial u}=0 \\
& t \frac{\partial E^{1}}{\partial t}-u \frac{\partial E^{1}}{\partial u}=-\frac{1}{6}\left(t^{2} \frac{\partial E^{0}}{\partial t}-2 t u \frac{\partial E^{0}}{\partial u}\right),
\end{aligned}
$$

Solving Equation (22) gives two functionally independent invariants:

$$
E_{1}=E_{1}^{0}(t, x, u)+\varepsilon E_{1}^{1}(t, x, u), \quad E_{2}=E_{2}^{0}(t, x, u)+\varepsilon E_{2}^{1}(t, x, u),
$$

for generator Equation (21).

The first equation in Equation (22) has two functionally independent solutions,

$$
E_{1}^{0}=x, \quad E_{2}^{0}=t u .
$$

Substituting $E_{1}^{0}=x$ into the second equation in Equation (22) and taking its simplest solution $E_{1}^{1}=0$, we obtain one invariant in Equation (23),

$$
E_{1}=x
$$

Now, we substitute the solution $E_{2}^{0}=t u$ of the first equation in Equation (22) into the second equation in Equation (22) and get a non-homogeneous linear equation:

$$
t \frac{\partial E_{1}^{1}}{\partial t}-u \frac{\partial E_{2}^{1}}{\partial u}=\frac{1}{6} t^{2} u
$$

The corresponding characteristic equation are:

$$
\frac{d t}{t}=-\frac{d u}{u}=6 \frac{d E_{2}^{1}}{t^{2} u}
$$

for which the first integral $t u=\lambda=$ const. We obtain:

$$
E_{2}^{1}=\frac{1}{6} t^{2} u+c
$$

Assuming $t=0$, we get the second invariant in Equation (23),

$$
E_{2}=t u+\frac{\varepsilon}{6} t^{2} u
$$

Note that invariants' Equations (24) and (25) are functionally independent. Letting $E_{2}=\phi\left(E_{1}\right)$, i.e.,

$$
\left(1+\frac{\varepsilon t}{6}\right) t u=\phi(x)
$$


and solving for $t u$ in the first order of precision,

$$
t u=\left(1+\frac{\varepsilon t}{6}\right)^{-1} \phi(x)=\left(1+\frac{\varepsilon t}{6}\right) \phi(x)+O(\varepsilon) .
$$

The approximately invariant solution is given by:

$$
u=\left(\frac{1}{t}-\frac{\varepsilon}{6}\right) \phi(x)
$$

From Equation (7), we obtain:

$$
\frac{d \phi}{d x}= \pm \sqrt{1+c \phi^{-4}}
$$

Setting $c=0$, we have $\phi(x)= \pm x$, and:

$$
u= \pm\left(\frac{x}{t}-\varepsilon \frac{x}{6}\right) .
$$

\section{Perturbed Nonlinear ( $2+1)$-Dimension Wave Equation}

Consider the perturbed nonlinear wave equation:

$$
u_{t t}+\varepsilon u_{t}=\left(g(u) u_{x}\right)_{x}+\left(h(u) u_{y}\right)_{y}
$$

where $\varepsilon$ is a small parameter. Putting $g(u)=h(u)=u$ gives:

$$
u_{t t}+\varepsilon u_{t}=\left(u u_{x}\right)_{x}+\left(u u_{y}\right)_{y} .
$$

The first method is used to obtain a complete approximate symmetry classification of Equation (28) with the first order of precision $o(\varepsilon)$. The approximate group generator of Equation (28) is of the form:

$$
\begin{aligned}
X & =X_{0}+\varepsilon X_{1} \\
& =\left(\tau_{0}+\varepsilon \tau_{1}\right) \frac{\partial}{\partial t}+\left(\xi_{0}+\varepsilon \xi_{1}\right) \frac{\partial}{\partial x}+\left(\theta_{0}+\varepsilon \theta_{1}\right) \frac{\partial}{\partial y}+\left(\eta_{0}+\varepsilon \eta_{1}\right) \frac{\partial}{\partial u},
\end{aligned}
$$

where $\tau_{i}, \xi_{i}, \theta_{i}$ and $\eta_{i}, i=0,1$, are unknown functions of $t, x, y$ and $u$.

\subsection{Exact Symmetries}

To find the exact symmetries, we solve the determining equation:

$$
\left.X_{0}^{(2)} F_{0}(z)\right|_{F_{0}(z)=0}=0,
$$

where $F_{0}(z)=u_{t t}-\left(u u_{x}\right)_{x}-\left(u u_{y}\right)_{y}$ is the unperturbed part of Equation (28) and $X_{0}^{(2)}$ is the second prolongation of the infinitesimal generator $X_{0}$ given by:

$$
\begin{aligned}
X_{0}^{(2)}=X_{0} & +\eta_{0}^{t} \frac{\partial}{\partial u_{t}}+\eta_{0}^{x} \frac{\partial}{\partial u_{x}}+\eta_{0}^{y} \frac{\partial}{\partial u_{y}}+\eta_{0}^{t t} \frac{\partial}{\partial u_{t t}}+\eta_{0}^{t x} \frac{\partial}{\partial u_{t x}} \\
& +\eta_{0}^{t y} \frac{\partial}{\partial u_{t y}}+\eta_{0}^{x x} \frac{\partial}{\partial u_{x x}}+\eta_{0}^{x y} \frac{\partial}{\partial u_{x y}}+\eta_{0}^{y y} \frac{\partial}{\partial u_{y y}} .
\end{aligned}
$$


Equation (30) takes the form:

$$
\begin{aligned}
\left(\eta_{0}\left(-u_{x x}-u_{y y}\right)\right. & +\eta_{0}^{x}\left(-2 u_{x}\right)+\eta_{0}^{y}\left(-2 u_{y}\right)+\eta_{0}^{x x}(-u) \\
& \left.+\eta_{0}^{y y}(-u)+\eta_{0}^{t t}\right)\left.\right|_{u_{t t}=\left(u u_{x}\right)_{x}+\left(u u_{y}\right)_{y}}=0
\end{aligned}
$$

where:

$$
\begin{aligned}
\eta_{0}^{x} & =D_{x} \eta_{0}-\left(u_{x} D_{x} \xi_{0}+u_{y} D_{x} \theta_{0}+u_{t} D_{x} \tau_{0}\right), \\
\eta_{0}^{y} & =D_{y} \eta_{0}-\left(u_{x} D_{y} \xi_{0}+u_{y} D_{y} \theta_{0}+u_{t} D_{y} \tau_{0}\right), \\
\eta_{0}^{t} & =D_{t} \eta_{0}-\left(u_{x} D_{t} \xi_{0}+u_{y} D_{t} \theta_{0}+u_{t} D_{t} \tau_{0}\right), \\
\eta_{0}^{x x} & =D_{x} \eta_{0}^{x}-u_{x x} D_{x} \xi_{0}-u_{x y} D_{x} \theta_{0}-u_{x t} D_{x} \tau_{0}, \\
\eta_{0}^{y y} & \left.=D_{y} \eta_{0}^{y}-u_{x y} D_{y} \xi_{0}-u_{y y} D_{y} \theta_{0}-u_{y t} D_{y} \tau_{0}\right), \\
\eta_{0}^{t t} & \left.=D_{t} \eta_{0}^{t}-u_{x t} D_{t} \xi_{0}-u_{y t} D_{t} \theta_{0}+u_{t t} D_{t} \tau_{0}\right) .
\end{aligned}
$$

Here, $D_{x}, D_{y}$ and $D_{t}$ denote the total derivative operators with respect to $x, y$ and $t$, respectively,

$$
\begin{aligned}
D_{x} & =\frac{\partial}{\partial x}+u_{x} \frac{\partial}{\partial u}+u_{x x} \frac{\partial}{\partial u_{x}}+u_{x y} \frac{\partial}{\partial u_{y}}+u_{x t} \frac{\partial}{\partial u_{t}}+\cdots+u_{x t t} \frac{\partial}{\partial u_{t t}} \\
D_{y} & =\frac{\partial}{\partial y}+u_{y} \frac{\partial}{\partial u}+u_{x y} \frac{\partial}{\partial u_{x}}+u_{y y} \frac{\partial}{\partial u_{y}}+u_{t y} \frac{\partial}{\partial u_{t}}+\cdots+u_{y t t} \frac{\partial}{\partial u_{t t}} \\
D_{t} & =\frac{\partial}{\partial t}+u_{t} \frac{\partial}{\partial u}+u_{x t} \frac{\partial}{\partial u_{x}}+u_{y t} \frac{\partial}{\partial u_{y}}+u_{t t} \frac{\partial}{\partial u_{t}}+\cdots+u_{t t t} \frac{\partial}{\partial u_{t t}}
\end{aligned}
$$

Equation (32) gives the following system of equations:

$$
\begin{aligned}
& \xi_{0 u}=0, \quad \xi_{0 t}=0, \quad \tau_{0 u}=0, \quad \tau_{0 y}=0, \quad \tau_{0 x}=0, \quad \theta_{0 u}=0, \quad \theta_{0 t}=0, \\
& \eta_{0 u u}=0, \quad-2 \eta_{0 x}-2 u \eta_{0 x u}+u \xi_{0 x x}+u \xi_{0 y y}=0, \quad-\eta_{0 u}+2 \xi_{0 x}-2 \tau_{0 t}=0, \\
& -2 \eta_{0 y}+u \theta_{0 x x}+u \theta_{0 y y}=0, \quad-2 \eta_{0 y}+2 \theta_{0 y}+\eta_{0_{u}}-2 \tau_{0 t}=0, \quad-u \eta_{0_{x x}}+\eta_{0_{t t}}-u \eta_{0_{y y}}=0, \\
& 2 \eta_{0 u t}-\tau_{0 t t}=0, \quad 2 u \xi_{0 x}-2 u \tau_{0 t}-\eta_{0}=0, \quad \theta_{0 x}+\xi_{0 y}=0, \quad-2 u \tau_{0 t}+2 u \theta_{0 y}-\eta_{0}=0
\end{aligned}
$$

Solving this system of PDEs, one has:

$$
\xi_{0}=a_{3} x+a_{1} y+a_{2}, \quad \theta_{0}=a_{3} y-a_{1} x+a_{6}, \quad \tau_{0}=a_{4} t+a_{5}, \quad \eta_{0}=2 u\left(a_{3}-a_{4}\right),
$$

where $a_{1}, a_{2}, a_{3}, a_{4}, a_{5}$ and $a_{6}$ are arbitrary constants. Hence, the infinitesimal generator for Equation (28) is:

$$
\begin{aligned}
X_{0}= & \left(a_{4} t+a_{5}\right) \frac{\partial}{\partial t}+\left(a_{3} x+a_{1} y+a_{2}\right) \frac{\partial}{\partial x} \\
& +\left(a_{3} y-a_{1} x+a_{6}\right) \frac{\partial}{\partial y}+\left(2 u\left(a_{3}-a_{4}\right)\right) \frac{\partial}{\partial u} .
\end{aligned}
$$

\subsection{Approximate Symmetries}

The auxiliary function $H$ is given by:

$$
H=\frac{1}{\varepsilon}\left[\left.X_{0}^{(k)}\left(F_{0}(z)+\varepsilon F_{1}(z)\right)\right|_{F_{0}(z)+\varepsilon F_{1}(z)=0}\right] .
$$


Substituting the generator $X_{0}$ into Equation (36) and:

$$
F_{0}(z)+\varepsilon F_{1}(z)=u_{t t}+\varepsilon u_{t}-\left(u u_{x}\right)_{x}-\left(u u_{y}\right)_{y}
$$

into Equation (37), we obtain:

$$
H=a_{4} u_{t}
$$

Now, we calculate operator $X_{1}$ by solving the inhomogeneous determining equation:

$$
\left.X_{1}^{(2)} F_{0}(z)\right|_{F_{0}(z)}+H=0,
$$

which can be written as:

$$
\left[\left.X_{1}^{(2)}\left(u_{t t}-\left(u u_{x}\right)_{x}-\left(u u_{y}\right)_{y}\right)\right|_{u_{t t}=\left(u u_{x}\right)_{x}+\left(u u_{y}\right)_{y}}\right]+a_{4} u_{t}=0 .
$$

Equation (39) generates the following system of equations:

$$
\begin{aligned}
& \xi_{1 u}=0, \quad \xi_{1 t}=0, \quad \tau_{1 u}=0, \quad \tau_{1 y}=0, \quad \tau_{1 x}=0, \quad \theta_{1 u}=0, \\
& \theta_{1 t}=0, \quad \eta_{1_{u u}}=0, \quad-2 \eta_{1} x-2 u \eta_{1_{x u}}+u \xi_{1 x x}+u \xi_{1 y y}=0, \quad-\eta_{1_{u}}+2 \xi_{1 x}-2 \tau_{1 t}=0, \\
& -2 \eta_{1_{y}}+u \theta_{1 x x}+u \theta_{1 y y}=0, \quad-2 \eta_{1_{y}}+2 \theta_{1 y}+\eta_{1_{u}}-2 \tau_{1 t}=0, \quad-u \eta_{1_{x x}}+\eta_{1_{t t}}-u \eta_{1_{y y}}=0, \\
& 2 \eta_{1_{u t}}-\tau_{1 t t}+a_{4}=0, \quad 2 u \eta_{1_{x}}-2 u \tau_{1 t}-\eta_{1}=0, \quad \theta_{1 x}+\xi_{1 y}=0, \quad-2 u \tau_{1 t}+2 u \theta_{1 y}-\eta_{1}=0 .
\end{aligned}
$$

Solving this system of PDEs, we obtain:

$$
\begin{array}{ll}
\xi_{1}=b_{3} x+b_{1} y+b_{2}, & \theta_{1}=b_{3} y-b_{1} x+b_{6}, \\
\tau_{1}=\frac{a_{4} t^{2}}{10}+b_{4} t+b_{5}, & \eta_{1}=2 u\left(b_{3}-\frac{1}{5} a_{4} t-b_{4}\right),
\end{array}
$$

where $b_{1}, b_{2}, b_{3}, b_{4}, b_{5}$ and $b_{6}$ are arbitrary constants. Thus, the approximate symmetries of Equation (28) are:

$$
\begin{aligned}
& X_{1}=y \frac{\partial}{\partial x}-x \frac{\partial}{\partial y}, \quad X_{2}=\frac{\partial}{\partial x}, \quad X_{3}=x \frac{\partial}{\partial x}+y \frac{\partial}{\partial x}+y \frac{\partial}{\partial y}+2 u \frac{\partial}{\partial u}, \\
& X_{4}=t \frac{\partial}{\partial t}-2 u \frac{\partial}{\partial u}+\varepsilon\left(\frac{t^{2}}{10} \frac{\partial}{\partial t}-\frac{2}{5} t u \frac{\partial}{\partial u}\right), \\
& X_{5}=\frac{\partial}{\partial t}, \quad X_{6}=\frac{\partial}{\partial y}, \quad X_{7}=\varepsilon X_{1}, \quad X_{8}=\varepsilon X_{2}, \\
& X_{9}=\varepsilon X_{3}, \quad X_{10}=\varepsilon X_{5}, \quad X_{11}=\varepsilon X_{6}, \quad X_{12}=\varepsilon X_{4} .
\end{aligned}
$$

\section{Remark 1.}

$$
X_{12}=\varepsilon\left(t \frac{\partial}{\partial t}-2 u t \frac{\partial}{\partial u}\right)
$$

In Table 2, we show that the previous generators span a twelve-dimensional approximate Lie algebra and, hence, generate a twelve-parameter approximate transformations group. 
Table 2. Approximate commutator table of approximate symmetries of the perturbed non-linear wave equation.

\begin{tabular}{ccccccccccccc}
\hline & $\boldsymbol{X}_{\mathbf{1}}$ & $\boldsymbol{X}_{\mathbf{2}}$ & $\boldsymbol{X}_{\mathbf{3}}$ & $\boldsymbol{X}_{\mathbf{4}}$ & $\boldsymbol{X}_{\mathbf{5}}$ & $\boldsymbol{X}_{\mathbf{6}}$ & $\boldsymbol{X}_{\mathbf{7}}$ & $\boldsymbol{X}_{\mathbf{8}}$ & $\boldsymbol{X}_{\mathbf{9}}$ & $\boldsymbol{X}_{\mathbf{1 0}}$ & $\boldsymbol{X}_{\mathbf{1 1}}$ & $\boldsymbol{X}_{\mathbf{1 2}}$ \\
\hline$X_{1}$ & 0 & $X_{6}$ & 0 & 0 & 0 & $-X_{2}$ & 0 & $X_{11}$ & 0 & 0 & 0 & 0 \\
$X_{2}$ & $-X_{6}$ & 0 & $X_{2}$ & 0 & 0 & 0 & $-X_{11}$ & 0 & $X_{8}$ & 0 & 0 & 0 \\
$X_{3}$ & 0 & $-X_{2}$ & 0 & 0 & 0 & $-X_{6}$ & 0 & $-X_{8}$ & 0 & 0 & $X_{11}$ & 0 \\
$X_{4}$ & 0 & 0 & 0 & 0 & $-X_{5}-\frac{2}{5} X_{12}$ & 0 & 0 & 0 & 0 & $-X_{10}$ & 0 & 0 \\
$X_{5}$ & 0 & 0 & 0 & $X_{5}+\frac{2}{5} X_{12}$ & 0 & 0 & 0 & 0 & 0 & 0 & 0 & $X_{10}$ \\
$X_{6}$ & $X_{2}$ & 0 & $X_{6}$ & 0 & 0 & 0 & $X_{8}$ & 0 & $X_{11}$ & 0 & 0 & 0 \\
$X_{7}$ & 0 & $X_{11}$ & 0 & 0 & 0 & $-X_{8}$ & 0 & 0 & 0 & 0 & 0 & 0 \\
$X_{8}$ & $-X_{11}$ & 0 & $X_{8}$ & 0 & 0 & 0 & 0 & 0 & 0 & 0 & 0 & 0 \\
$X_{9}$ & 0 & $-X_{8}$ & 0 & 0 & 0 & $-X_{11}$ & 0 & 0 & 0 & 0 & 0 & 0 \\
$X_{10}$ & 0 & 0 & 0 & $X_{10}$ & 0 & 0 & 0 & 0 & 0 & 0 & 0 & 0 \\
$X_{11}$ & 0 & 0 & $X_{11}$ & 0 & 0 & 0 & 0 & 0 & 0 & 0 & 0 & 0 \\
$X_{12}$ & 0 & 0 & 0 & 0 & $-X_{10}$ & 0 & 0 & 0 & 0 & 0 & 0 & 0 \\
\hline
\end{tabular}

\subsection{Approximate Invariant Solutions}

Reconsider Equation (28):

$$
u_{t t}-\varepsilon u_{t}=\left(u u_{x}\right)_{x}+\left(u u_{y}\right)_{y}
$$

and the symmetry:

$$
X_{4}=t \frac{\partial}{\partial t}-2 u \frac{\partial}{\partial u}+\frac{\varepsilon}{10}\left(t^{2} \frac{\partial}{\partial t}-4 u t \frac{\partial}{\partial u}\right) .
$$

The approximate invariant for Equation (42) is of the form:

$$
E(t, x, y, u, \varepsilon)=E^{0}(t, x, y, u)+\varepsilon E^{1}(t, x, y, u)+o(\varepsilon),
$$

determined by the equation $X(E)=o(\varepsilon)$. Using the notation:

$$
X=X^{0}+\varepsilon X^{1}
$$

where:

$$
X^{0}=t \frac{\partial}{\partial t}-2 u \frac{\partial}{\partial u}, \quad X^{1}=\frac{1}{10}\left(t^{2} \frac{\partial}{\partial t}-4 t u \frac{\partial}{\partial u}\right) ;
$$

for operator Equation (42), we write the determining equation $X(E)=o(\varepsilon)$ for the approximate invariants in the form:

$$
\begin{aligned}
& X^{0}\left(E^{0}\right)+\varepsilon\left[X^{0}\left(E^{1}\right)+X^{1}\left(E^{0}\right)\right]=0, \quad X^{0}\left(E^{0}\right)=0, \\
& X^{0}\left(E^{1}\right)+X^{1}\left(E^{0}\right)=0
\end{aligned}
$$

or:

$$
\begin{aligned}
& t \frac{\partial E^{0}}{\partial t}-2 u \frac{\partial E^{0}}{\partial u}=0 \\
& t \frac{\partial E^{1}}{\partial t}-2 u \frac{\partial E^{1}}{\partial u}=-\frac{1}{10}\left(t^{2} \frac{\partial E^{0}}{\partial t}-4 t u \frac{\partial E^{0}}{\partial u}\right)
\end{aligned}
$$

Solving Equation (43) gives two functionally independent invariants:

$$
E_{1}=E_{1}^{0}(t, x, y, u)+\varepsilon E_{1}^{1}(t, x, y, u), \quad E_{2}=E_{2}^{0}(t, x, y, u)+\varepsilon E_{2}^{1}(t, x, y, u),
$$

for generator Equation (42). 
The first equation in Equation (43) has two functionally independent solutions:

$$
E_{1}^{0}=x y, \quad E_{2}^{0}=t^{2} u
$$

Substituting $E_{1}^{0}=x y$ into the second equation in Equation (43) and taking its simplest solution $E_{1}^{1}=0$, we obtain one invariant in Equation (44),

$$
E_{1}=x y
$$

Note that the dependent variable $u$ does not appear in Equation (45). Now, we substitute the solution $E_{2}^{0}=t^{2} u$ of the first equation in Equation (43) into the second equation in Equation (43) and obtain non-homogeneous linear equation:

$$
t \frac{\partial E_{1}^{1}}{\partial t}-2 u \frac{\partial E_{2}^{1}}{\partial u}=\frac{1}{5}\left(t^{3} u\right)
$$

The corresponding characteristic equations are:

$$
\frac{d t}{t}=-\frac{d u}{2 u}=5 \frac{d E_{2}^{1}}{t^{2} u}
$$

with the first integral $t^{2} u=\lambda=$ const. Therefore, the second equation:

$$
\frac{d t}{t}=5 \frac{d E_{2}^{1}}{t^{3} u}
$$

gives:

$$
E_{2}^{1}=\frac{1}{5} t^{3} u+c
$$

Assuming that $c=0$, we obtain the second invariant in Equation (44),

$$
E_{2}=t^{2} u+\frac{\varepsilon}{5} t^{3} u
$$

Note that $E_{1}$ and $E_{2}$ are functionally independent. Letting $E_{2}=\psi(E 1)$, i.e.,

$$
\left(t^{2} u+\frac{\varepsilon}{5} t^{3} u\right)=\psi(x y)
$$

and solving for $t^{2} u$ in the first order of precision,

$$
t^{2} u=\left(1+\frac{\varepsilon}{5} t\right)^{-1} \phi(x y)=\left(1-\frac{\varepsilon}{5} t\right) \phi(x y)+o(\varepsilon),
$$

yield the approximate invariant solution:

$$
u(t, x, y)=\left(\frac{1}{t^{2}}-\frac{\varepsilon}{5 t}\right) \phi(x y) .
$$

From Equation (28), we obtain:

$$
\left(\frac{\partial \phi}{\partial x}\right)^{2}+\frac{\partial^{2} \phi}{\partial x^{2}} \cdot \phi+\left(\frac{\partial \phi}{\partial y}\right)^{2}+\frac{\partial^{2} \phi}{\partial y^{2}} \cdot \phi-6 \phi=0 .
$$

Case I: Let $\psi(x y)$ be of the form $\phi(x y)=(x y)^{\alpha}$. From Equation (49), one obtains:

$$
\left[2 \alpha^{2}-\alpha\right]\left(x^{\alpha-2} y^{\alpha}+x^{\alpha} y^{\alpha-2}\right)-6=0
$$


For $\alpha=2$, we have:

$$
u(t, x, y)=\left(\frac{1}{t^{2}}-\frac{\varepsilon}{5 t}\right)\left(x^{2} y^{2}\right) \text { s.t } x^{2}+y^{2}=1
$$

An approximate solution for this case is depicted in Figure 1.

Case II: Let $\phi(x y)=A(x) B(x)$. Equation (49) gives the following equation:

$$
B\left(\frac{A^{\prime 2}+A A^{\prime \prime}}{A}\right)+A\left(\frac{B^{\prime 2}+B B^{\prime \prime}}{B}\right)-6=0
$$

where:

$$
A^{\prime}=\frac{\partial A}{\partial x}, \quad A^{\prime \prime}=\frac{\partial^{2} A}{\partial x^{2}}, \quad B^{\prime}=\frac{\partial B}{\partial y}, \quad B^{\prime \prime}=\frac{\partial^{2} B}{\partial y^{2}}
$$

Equation (50) leads to the following ordinary differential equations:

$$
A A^{\prime \prime}+A^{\prime 2}-d_{1} A^{2}-c_{1} A=0,
$$

and:

$$
B B^{\prime \prime}+B^{\prime 2}-d_{2} B^{2}-c_{2} B=0,
$$

where $c_{1}, c_{2}, d_{1}, d_{2}$ are constants.

Let $\gamma(x)=A^{2}(x)$. From Equations (51) and (52), we obtain:

$$
\left(2 \ln \left(d_{1} A(x)+c_{1}\right)-1\right)\left(\frac{A^{2}(x)}{2 d_{1}}-\frac{c_{1}^{2}}{2 d_{1}^{2}}\right)+\frac{c_{1}}{d_{1}^{2}} A^{2}(x)=\frac{x^{2}}{2}+c_{3} x+c_{4},
$$

and:

$$
\left(2 \ln \left(d_{2} B(x)+c_{2}\right)-1\right)\left(\frac{B^{2}(x)}{2 d_{2}}-\frac{c_{2}^{2}}{2 d_{2}^{2}}\right)+\frac{c_{2}}{d_{2}^{2}} B^{2}(x)=\frac{x^{2}}{2}+c_{5} x+c_{6},
$$

where $c_{3}, c_{4}, c_{5}, c_{6}$ are arbitrary constants. Therefore, a solution in this case is of the form:

$$
u(t, x, y)=\left(\frac{1}{t^{2}}-\frac{\varepsilon}{5 t}\right) A(x) B(x)
$$

We plot an approximate solution for this case in Figure 2.

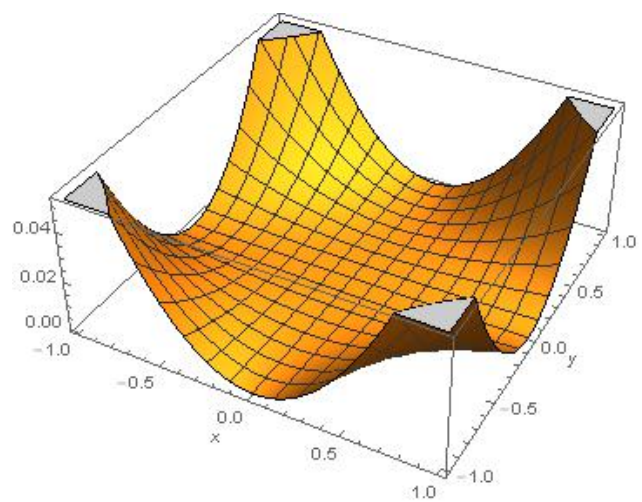

Figure 1. CaseI: approximate invariant solution of Equation (28) for $t=\pi,-1 \leq x \leq 1,-1 \leq y \leq 1$, $\varepsilon=0.1$. 


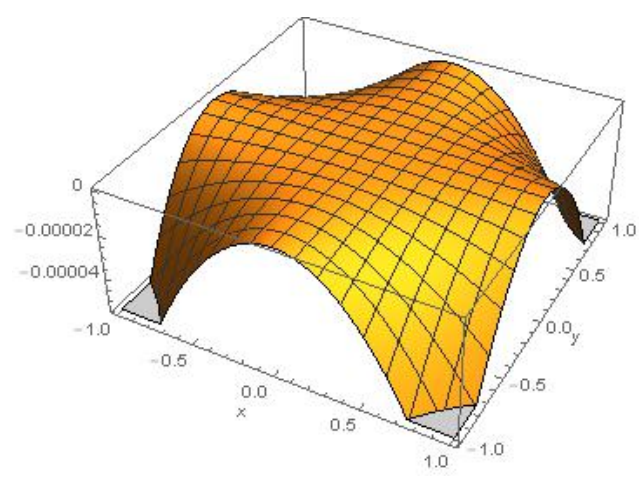

Figure 2. Case II:approximate invariant solution of Equation (28) for $t=100,-1 \leq x \leq 1,-1 \leq y \leq 1$, $\varepsilon=0.1$.

\section{Nonlinear Wave Equation with a Forcing Term}

In this section, we discuss the nonlinear $(2+1)$-dimensional wave equation with a forcing term:

$$
u_{t t}-\left(u u_{x}\right)_{x}-\left(u u_{y}\right)_{y}=\varepsilon f(u) .
$$

\subsection{Approximate Symmetries by Method I}

Exact symmetries of the unperturbed part $(\varepsilon=0)$ of Equation (56) are given by:

$$
\xi_{0}=a_{3} x+a_{1} y+a_{2}, \quad \theta_{0}=a_{3} y-a_{1} x+a_{6}, \quad \tau_{0}=a_{4} t+a_{5}, \quad \eta_{0}=2 u\left(a_{3}-a_{4}\right),
$$

where $a_{1}, a_{2}, a_{3}, a_{4}, a_{5}$ and $a_{6}$ are arbitrary constants.

Consider the auxiliary function:

$$
H=\frac{1}{\varepsilon}\left[\left.X^{0}\left(F_{0}(z)+\varepsilon F_{1}(z)\right)\right|_{F_{0}(z)+\varepsilon F_{1}(z)=0^{\prime}}\right.
$$

where:

$$
X^{0}=\tau_{0} \frac{\partial}{\partial t}+\xi_{0} \frac{\partial}{\partial x}+\theta_{0} \frac{\partial}{\partial y}+\eta_{0} \frac{\partial}{\partial u} .
$$

Using Equations (56) and (57), one obtains:

$$
H=-2 a_{3}\left(u f^{\prime}(u)+f(u)\right)+2 a_{4}\left(u f^{\prime}(u)-2 f(u)\right) .
$$

Now we calculate the operator $X_{1}$ with the condition that

$$
\left.X^{1}\left(F_{0}(z)\right)\right|_{F_{0}(z)=0}+H=0 .
$$

Condition Equation (60) can be written as:

$$
\begin{array}{r}
{\left[\left.X_{1}^{(2)}\left(u_{t t}-\left(u u_{x}\right)_{x}-\left(u u_{y}\right)_{y}\right)\right|_{u_{t t}=\left(u u_{x}\right)_{x}+\left(u u_{y}\right)_{y}}\right]-2 c_{3}\left(u f^{\prime}(u)+f(u)\right)} \\
+2 c_{4}\left(u f^{\prime}(u)-2 f(u)\right)=0,
\end{array}
$$

where $X_{1}^{(2)}$ is the second prolongation of $X_{1}$. 
Equation (61) yields the following system of equations:

$$
\begin{aligned}
& \xi_{1 u}=0, \quad \xi_{1 t}=0, \quad \tau_{1 u}=0, \quad \tau_{1 y}=0, \quad \tau_{1 x}=0, \\
& \theta_{1 u}=0, \quad \theta_{1 t}=0, \quad \eta_{1 u u}=0, \quad-2 \eta_{1 x}-2 u \eta_{1_{x u}}+u \xi_{1 x x}+u \xi_{1 y y}=0, \\
& -\eta_{1_{u}}+2 \xi_{1 x}-2 \tau_{1 t}=0 \quad-2 \eta_{1_{y}}+u \theta_{1 x x}+u \theta_{1 y y}=0, \quad-2 \eta_{1_{y}}+2 \theta_{1 y}+\eta_{1_{u}}-2 \tau_{1 t}=0, \\
& -u \eta_{1_{x x}}+\eta_{1_{t t}}-u \eta_{1_{y y}}-2 a_{3}\left(u f^{\prime}(u)+f(u)\right)=0, \quad 2 \eta_{1_{u t}}-\tau_{1 t t}=0, \\
& 2 u \xi_{1 x}-2 u \tau_{1 t}-\eta_{1}=0, \quad \theta_{1 x}+\xi_{1 y}=0, \quad-2 u \tau_{1 t}+2 u \theta_{1 y}-\eta_{1}=0,
\end{aligned}
$$

Solving this system of PDEs, we obtain:

$$
\begin{aligned}
& \xi_{1}=b_{3} x+b_{1} y+b_{2}, \quad \theta_{1}=b_{3} y-b_{1} x+b_{6}, \\
& \tau_{1}=b_{4} t+b_{5}, \quad \eta_{1}=2 u\left(b_{3}-b_{4}\right),
\end{aligned}
$$

where $b_{1}, b_{2}, b_{3}, b_{4}, b_{5}$ and $b_{6}$ are arbitrary constants.

Case I: $a_{3}=0$. The scaling operator:

$$
X_{3}=x \frac{\partial}{\partial x}+y \frac{\partial}{\partial y}+2 u \frac{\partial}{\partial u}
$$

is not stable, and hence, Equation (56) does not inherit symmetries of its unperturbed part.

Case II: Solving the first order linear differential equation $u f^{\prime}(u)+f(u)=0$, we obtain $f(u)=k_{1} / u$, where $k_{1}$ is a constant. The approximate symmetry generator of Equation (56) is given by:

$$
\begin{aligned}
X= & X_{0}+\varepsilon X_{1} \\
= & {\left[\left(a_{3}+\varepsilon b_{3}\right) x+\left(a_{1}+\varepsilon b_{1}\right) y+\left(a 2+\varepsilon b_{2}\right)\right] \frac{\partial}{\partial x} } \\
& +\left[\left(a_{3}+\varepsilon b_{3}\right) y-\left(a_{1}+\varepsilon b_{1}\right) x+\left(a_{6}+\varepsilon b_{6}\right)\right] \frac{\partial}{\partial y} \\
& +\left[\left(a_{4}+\varepsilon b_{4}\right) t+\left(a_{5}+\varepsilon b_{5}\right)\right] \frac{\partial}{\partial t}+\left[2 u\left(\left(a_{3}+\varepsilon b_{3}\right)-\left(a_{4}+\varepsilon b_{4}\right)\right)\right] \frac{\partial}{\partial u}
\end{aligned}
$$

These additional symmetries are actually the same as those obtained from the unperturbed equation that are considered as trivial symmetries. To summarize: in this case, Method I only gives trivial symmetries.

\subsection{Approximate Symmetries by Method II}

We expand the dependent variable to the first order of $\varepsilon$ as follows:

$$
u=v+\varepsilon w+o(\varepsilon), \quad \varepsilon \rightarrow 0 .
$$

Taylor expansion of $f$ in the first order of precision is given by:

$$
f(u)=\frac{k_{1}}{v}\left(1-\frac{\varepsilon w}{v}+o(\varepsilon)\right)=\frac{k_{1}}{v}-\frac{\varepsilon k_{1} w}{v^{2}}+o(\varepsilon), \quad \varepsilon \rightarrow 0 .
$$

Substituting the above expansion into Equation (56) and separating at each order of perturbation parameter, one may obtain:

$$
\begin{aligned}
v_{t t}-v_{x}^{2}-v v_{x x}-v_{y}^{2}-v v_{y y} & =0 \\
w_{t t}-2 v_{x} w_{x}-v w_{x x}-w v_{x x}-2 v_{y} w_{y}-v w_{y y}-w v_{y y} & =\frac{k_{1}}{v} .
\end{aligned}
$$


Now, the infinitesimal generator for the problem is:

$$
\begin{aligned}
X=\tau(t, x, y, v, w) \frac{\partial}{\partial t} & +\xi(t, x, y, v, w) \frac{\partial}{\partial x}+\theta(t, x, y, v, w) \frac{\partial}{\partial y} \\
& +\phi(t, x, y, v, w) \frac{\partial}{\partial v}+\eta(t, x, y, v, w) \frac{\partial}{\partial w} .
\end{aligned}
$$

Using standard Lie group analysis, we obtain the infinitesimals as follows:

$$
\begin{aligned}
& \tau=c_{4} t+c_{5}, \quad \xi=c_{1} x-c_{3} y+c_{6}, \quad \theta=c_{3} x+c_{1} y+c_{2}, \\
& \phi=2 v\left(-c_{4}+c_{1}\right), \quad \eta=-2 w\left(c_{1}-2 c_{4}\right),
\end{aligned}
$$

where $c_{1}, c_{2}, c_{3}, c_{4}, c_{5}$ and $c_{6}$ are arbitrary constants. Hence, we have the following symmetries:

$$
\begin{aligned}
& X_{1}=x \frac{\partial}{\partial x}+y \frac{\partial}{\partial y}+2 v \frac{\partial}{\partial v}-2 w \frac{\partial}{\partial v}, \quad X_{2}=\frac{\partial}{\partial y^{\prime}}, \quad X_{3}=-y \frac{\partial}{\partial x}+x \frac{\partial}{\partial y^{\prime}} \\
& X_{4}=t \frac{\partial}{\partial t}-2 v \frac{\partial}{\partial v}+4 w \frac{\partial}{\partial w}, \quad X_{5}=\frac{\partial}{\partial t^{\prime}}, \quad X_{6}=\frac{\partial}{\partial x} .
\end{aligned}
$$

Table 3 shows that Equation (67) spans a sixth-dimensional Lie algebra.

Table 3. Commutators span six-dimensional Lie algebra.

\begin{tabular}{ccccccc}
\hline & $X_{1}$ & $X_{2}$ & $X_{3}$ & $X_{4}$ & $X_{5}$ & $X_{6}$ \\
\hline$X_{1}$ & 0 & $-X_{2}$ & 0 & 0 & 0 & $-X_{6}$ \\
$X_{2}$ & $X_{2}$ & 0 & $-X_{6}$ & 0 & 0 & 0 \\
$X_{3}$ & 0 & $X_{6}$ & 0 & 0 & 0 & $-X_{2}$ \\
$X_{4}$ & 0 & 0 & 0 & 0 & $-X_{5}$ & 0 \\
$X_{5}$ & 0 & 0 & 0 & $X_{5}$ & 0 & 0 \\
$X_{6}$ & $X_{6}$ & 0 & $X_{2}$ & 0 & 0 & 0 \\
\hline
\end{tabular}

\subsection{Approximate Invariant Solution}

Using $X_{3}$ from Equation (67), we retrieve the following characteristic equations:

$$
\frac{d x}{-y}=\frac{d y}{x}=\frac{d v}{0}=\frac{d w}{0} .
$$

The equations in Equation (68) yield $\alpha=x^{2}+y^{2}$ and suggest that $w=w(\alpha), v=v(\alpha)$. Derivatives of dependent variables $v$ and $w$ with respect to $x$ and $y$ are:

$$
\begin{aligned}
& v_{t}=v_{\alpha} \frac{\partial \alpha}{\partial t}=0, \quad v_{t t}=0 \\
& v_{x}=v-\alpha \frac{\partial \alpha}{\partial x}=2 x v_{\alpha}, \quad v_{x x}=2 v_{\alpha}+2 x v_{\alpha \alpha} \frac{\partial \alpha}{\partial x}=2 v_{\alpha}+4 x^{2} v_{\alpha \alpha} \\
& v_{y}=v-\alpha \frac{\partial \alpha}{\partial y}=2 y v_{\alpha}, \quad v_{y y}=2 v_{\alpha}+2 y v_{\alpha \alpha} \frac{\partial \alpha}{\partial y}=2 v_{\alpha}+4 y^{2} v_{\alpha \alpha} \\
& w_{t}=w_{\alpha} \frac{\partial \alpha}{\partial t}=0, \quad w_{t t}=0 \\
& w_{x}=w-\alpha \frac{\partial \alpha}{\partial x}=2 x w_{\alpha}, \quad w_{x x}=2 w_{\alpha}+2 x w_{\alpha \alpha} \frac{\partial \alpha}{\partial x}=2 w_{\alpha}+4 x^{2} w_{\alpha \alpha} \\
& w_{y}=w-\alpha \frac{\partial \alpha}{\partial y}=2 y w_{\alpha}, \quad w_{y y}=2 w_{\alpha}+2 y w_{\alpha \alpha} \frac{\partial \alpha}{\partial y}=2 w_{\alpha}+4 y^{2} w_{\alpha \alpha} .
\end{aligned}
$$


These equations lead to the following second order ordinary differential equations:

$$
\begin{aligned}
\alpha v_{\alpha}^{2}+\alpha v v_{\alpha \alpha}+v v_{\alpha} & =0, \\
2 \alpha v_{\alpha} w_{\alpha}+v w_{\alpha}+\alpha v w_{\alpha \alpha}+w v_{\alpha}+\alpha w v_{\alpha \alpha} & =\frac{k_{1}}{4 v} .
\end{aligned}
$$

Substituting:

$$
v(\alpha)=\frac{H(\alpha)^{2}}{2}
$$

into the first equation of Equation (69), we obtain:

$$
\frac{\partial H(\alpha)}{\partial \alpha}+\alpha \frac{\partial^{2} H(\alpha)}{\partial \alpha^{2}}=0
$$

We have $H(\alpha)=c_{1} \ln \alpha+c_{2}$, where $c_{1}$ and $c_{2}$ are arbitrary constants of the integration. Thus, $v(\alpha)=\sqrt{c_{2}+c_{1} \ln \alpha}$. Put $c_{2}=0, c_{1}=1$. The second equation of Equation (69) is reduced to the following second-order ordinary differential equation:

$$
(1+\ln \alpha) w_{\alpha}+(\alpha \ln \alpha) w_{\alpha \alpha}-\frac{1}{4 \ln \alpha} w=\frac{k_{1}}{4} .
$$

Observe that it is not straight forward to obtain a solution for Equation (70). However, we may obtain an asymptotic estimate of the solution of Equation (70) using the asymptotic expansions [22].

Definition 1. The function $f(x)=O(g(x))$ as $x \rightarrow x_{0}$ if there exists a constant $C$ such that $\lim _{x \rightarrow x_{0}} f / g=C$.

In Equation (70), we have $(1+\ln \alpha)=O(\alpha),\left(\alpha \ln \alpha^{2}\right)=O\left(\alpha^{2}\right)$ and $\frac{1}{4 \ln \alpha}=O(1)$ as $\alpha \rightarrow \infty$. For large values of $\alpha$, Equation (70) is asymptotically equivalent to the following equation:

$$
\alpha^{2} w_{\alpha \alpha}+\alpha w_{\alpha}+w=\frac{k_{1}}{4} .
$$

The solution of the above non-homogeneous Cauchy-Euler equation is:

$$
w(\alpha)=k_{2} \sin (\ln \alpha)+k_{3} \cos (\ln \alpha)+\frac{k_{1}}{4}
$$

where $k_{2}, k_{3}$ are constants. Lastly, we re-cast the solution in original coordinates as:

$$
\begin{aligned}
u(t, x, y) & =v(t, x, y)+\varepsilon w(t, x, y) \\
& =\sqrt{\ln \left(x^{2}+y^{2}\right)}+\varepsilon\left(k_{2} \sin \left(\ln \left(x^{2}+y^{2}\right)\right)+k_{3} \cos \left(\ln \left(x^{2}+y^{2}\right)\right)+\frac{k_{1}}{4}\right) .
\end{aligned}
$$

This is an approximate solution invariant under rotation in $x-y$, dilation in space and $u$ coordinates. We depict an invariant solution for the unperturbed equation in Figure 3 and an approximate solution of the perturbed one in Figure 4. 


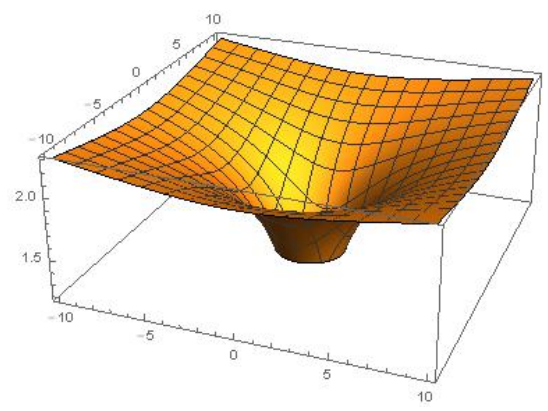

Figure 3. Invariant solution of the unperturbed equation of Equation (56) for $-10 \leq x \leq 10$, $-10 \leq y \leq 10, x^{2}+y^{2} \geq 1$

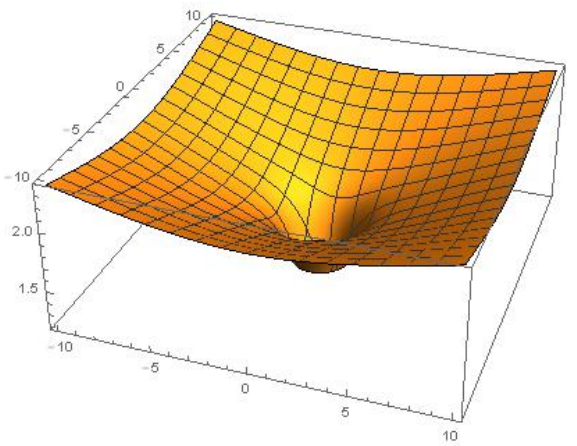

Figure 4. Approximate invariant solution of Equation (56) for $-10 \leq x \leq 10,-10 \leq y \leq 10$, $x^{2}+y^{2} \geq 1, k_{1}=4, k_{2}=1, k 3=1, \varepsilon=0.1$.

\section{Concluding Remarks}

In this work, we have studied a class of perturbed nonlinear wave equations via Lie symmetry analysis. Two methods have been employed to obtain approximate symmetries used to construct invariant solutions of the equations. There was a case where Method I gives only trivial solutions. We applied Method II to this case and obtained the invariant solutions of the equation. Many problems arising from physical or engineering situations may be dealt with by approximate Lie symmetry analysis. We plan to investigate modified and perturbed forms of Korteweg-de Vries (KdV) equations using this approach.

Acknowledgments: The authors are grateful to King Fahd University of Petroleum \& Minerals for supporting the research.

Author Contributions: Waheed: working out symmetries and approximation. Zaman: proposing, guiding and leading. Saleh: working out plan, verifying, writing up and final form.

Conflicts of Interest: The authors declare no conflict of interest.

\section{References}

1. Baikov, V.A.; Gazizov, R.K.; Ibragimov, N. Approximate symmetries. Mat. Sb. 1988, 178, 435-450.

2. Fushchich, W.; Shtelen, W. On approximate symmetry and approximate solutions of the nonlinear wave equation with a small parameter. J. Phys. A Math. Gen. 1989, 22, L887.

3. Gazizov, R.K. Lie algebras of approximate symmetries. J. Nonlinear Math. Phys. 1996, 3, 96-101.

4. Bokhari, A.H.; Kara, A.; Zaman, F.D. Invariant solutions of certain nonlinear evolution type equations with small parameters. Appl. Math. Comput. 2006, 182, 1075-1082.

5. Jefferson, G.; Carminati, J. Asp: Automated symbolic computation of approximate symmetries of differential equations. Comput. Phys. Commun. 2013, 184, 1045-1063. 
6. Li, X.; Qian, S. Approximate symmetry reduction to the perturbed coupled kdv equations derived from two-layer fluids. Math. Sci. 2012, 6, 13.

7. Zhang, Z.Y.; Chen, Y.F.; Yong, X.L. Classification and approximate solutions to a class of perturbed nonlinear wave equations. Commun. Theor. Phys. 2009, 52, 769-772.

8. Pakdemirli, M.; Yürüsoy, M.; Dolapci, I. Comparison of approximate symmetry methods for differential equations. Acta Appl. Math. 2004, 80, 243-271.

9. Valenti, A. Approximate symmetries for a model describing dissipative media. In Proceedings of the 10 th International Conference in Modern Group Analysis, Larnaca, Cyprus, 24-31 October 2004; Volume 236, p. 243.

10. Ma, W.X. A refined invariant subspace method and applications to evolution equations. Sci. China Math. 2012, 55, 1769-1778.

11. Zhang, Z.; Gao, B.; Chen, Y. Second-order approximate symmetry classification and optimal system of a class of perturbed nonlinear wave equations. Commun. Nonlinear Sci. Numer. Simul. 2011, 16, 2709-2719.

12. Zhang, Z.-Y.; Yong, X.-L.; Chen, Y.-F. A new method to obtain approximate symmetry of nonlinear evolution equation from perturbations. Chin. Phys. B 2009, 18, 2629-2633.

13. Baikov, V.; Gazizov, R.; Ibragimov, N. Approximate transformation groups and deformations of symmetry lie algebras. In CRC Handbook of Lie Group Analysis of Differential Equations; CRC Press: Boca Raton, FL, USA, 1996; Volume 3.

14. Grigotieve, Y.N.; Ibragimov, N.H.; Kovalev, V.F.; Meleshko, S.V. Symmetries of Integro-Differential Equations with Application in Mechanics and Plasma Physics; Springer: Berlin, Germany, 2010.

15. Bokhari, A.H.; Kara, A.; Karim, M.; Zaman, F.D. Invariance analysis and variational conservation laws for the wave equation on some manifolds. Int. J. Theor. Phys. 2009, 48, 1919-1928.

16. Ahmad, A.; Bokhari, A.H.; Kara, A.; Zaman, F.D. A complete symmetry classification and reduction of some classes of the nonlinear (1-2) wave equation. Quaest. Math. 2010, 33, 75-94.

17. Euler, M.; Euler, N.; Kohler, A. On the construction of approximate solutions for a multidimensional nonlinear heat equation. J. Phys. A Math. Gen. 1994, 27, 2083-2092.

18. Euler, N.; Euler, M. Symmetry properties of the approximations of multidimensional generalized van der pol equations. J. Nonlinear Math. Phys. 1994, 1, 41-59.

19. Ma, W.X.; Fuchssteiner, B. Integrable theory of the perturbation equations. Chaos Soliton Fractal 1996, 7, 1227-1250.

20. Ma, W.X. A bi-Hamiltonian formulation for triangular systems by perturbations. J. Math. Phys. 2002, 43, 1408-1421.

21. Ibragimov, N.H.; Kovalev, V.F. Approximate and Renormgroup Symmetries; Springer: Berlin, Germany, 2009.

22. Murray, J.D. Asymptotic analysi. In Applied Mathematical Sciences; Springer: New York, NY, USA, 1984; Volume 1.

(C) 2017 by the authors. Licensee MDPI, Basel, Switzerland. This article is an open access article distributed under the terms and conditions of the Creative Commons Attribution (CC BY) license (http:/ / creativecommons.org/licenses/by/4.0/). 